\section{Research Square}

Preprints are preliminary reports that have not undergone peer review.

They should not be considered conclusive, used to inform clinical practice, or referenced by the media as validated information.

\title{
PD-1, TIM-3 and LAG-3 Expression in T Cells in a Patient with Recurrent Ossifying Fibroma. A Case Report.
}

\section{Alejandro García-Muñoz}

Universidad Nacional Autonoma de Mexico

Nayeli Goreti Nieto-Velázquez

Hospital Juarez de Mexico

Gabriela Damian-Morales

Hospital Juarez de Mexico

Carlos Liceaga-Escalera

Hospital Juarez de Mexico

Luis Alberto Montoya-Perez

Hospital Juarez de Mexico

Madeleine Cruz-Vélez

Hospital Juarez de Mexico

Pabel Antonio Gómez-Hernández

Hospital Juarez de Mexico

Cynthia Trejo-Iriarte

Hospital Juarez de Mexico

Rodolfo Pastelin-Palacios

Universidad Nacional Autonoma de Mexico

Mario Adán Moreno-Eutimio ( $\square$ marioadan@inmunoquimica.com )

Universidad Nacional Autonoma de Mexico https://orcid.org/0000-0002-9955-8498

\section{Case Report}

Keywords: ossifying fibroma, exhausted T cell, and odontogenic tumors

Posted Date: July 23rd, 2020

DOI: https://doi.org/10.21203/rs.3.rs-45451/v1

License: (1) (1) This work is licensed under a Creative Commons Attribution 4.0 International License.

Read Full License 


\section{Abstract}

Background: Central ossifying fibroma is a benign, slow-growing tumor of mesenchymal origin with a predilection for the mandibular premolar and molar areas. The immunophenotype of $\mathrm{T}$ cells involved in the antitumor response against this benign tumor is unknown.

Case presentation: In this case report, we described a case of a 48-year-old woman presenting with a very large recurrent ossifying fibroma in the mandible, which was successfully treated with hemimaxillectomy. In addition, we evaluated the expression of programmed cell death protein-1 (PD-1), lymphocyte activation gene-3 (LAG-3), T cell immunoglobulin and mucin-domain containing-3 (TIM-3), cytotoxic T lymphocyte-associated antigen-4 (CTLA-4), CD69 (activation inducer molecule) and CD25 (a chain of the high-affinity IL-2 receptor) in T cell populations from the tumor and peripheral blood of this uncommon lesion.

Conclusions: The patient presented recurrent ossifying fibroma, and the tumor-infiltrating and peripheral blood T cells showed expression of PD-1, LAG-3, and TIM-3, suggesting an exhausted T cell response.

\section{Background}

According to the 2017 WHO classification, there are three variants of ossifying fibromas: cementoossifying fibroma, juvenile trabecular ossifying fibroma, and juvenile psammomatoid ossifying fibroma (1). In general, ossifying fibroma (OF) is a well-demarcated lesion composed of fibrous connective tissue with varying degrees of cellularity and calcification areas. The calcified structures consist of rounded or lobulated basophilic masses (cementum-like), trabeculae of osteoid or bone, or combinations of the two. Due to the presence of this cementum-like material, OF has also been called cementifying fibroma or cemento-ossifying fibroma (2). Clinically, OF is a slowly progressing tumor of craniofacial bones, with a tendency towards locally aggressive behavior and recurrence. This tumor usually shows bone expansion, is painless, shows no cortical perforation or tooth root resorption, and has a mixed unilocular radiodensity appearance and well-defined boundaries on radiological examination (3).

The adaptive antitumor immune response begins with professional antigen-presenting cells that capture tumor antigens and process and present them on the cell surface in complexes with major histocompatibility complex (MHC) class I and II molecules to naive T cells. This interaction results in the maturation and activation of tumor-specific cytotoxic T (Tc) lymphocytes capable of migrating to tumor sites, identifying tumor cells, and destroying them. Additionally, the interaction triggers a response by $T$ helper (Th) cells, which stimulate the $T$ and $B$ cell arms of the antitumor immune response (4). Studies about infiltrating immune cells in OF or odontogenic tumors are limited.

Tumor cells evade the immune response and progress through different mechanisms (5), including activation of immune checkpoint pathways that suppress antitumor immune responses. Cancer immunotherapy with immune checkpoint blockade has been proven to be effective in the treatment of many tumors, such as melanoma and non-small-cell lung carcinoma (6). The immune checkpoint targets 
include molecules on T lymphocytes, such as programmed cell death protein 1 (PD-1) (7), lymphocyte activation gene-3 (LAG-3) (8), T cell immunoglobulin and mucin-domain containing-3 (TIM-3) (9), and cytotoxic T lymphocyte-associated antigen-4 (CTLA-4) (10), among others. Thus far, there are no studies that have explored the expression of these molecules in OF; immunotherapy by immune checkpoint blockade may be useful in OF.

\section{Case Presentation}

At the maxillofacial service at Hospital Juárez de México, a 48-year-old female who had undergone surgery three years prior presented with a benign fibro-osseous lesion. The first treatment was enucleation, and the second treatment was remodeling. The final pathology results reported OF. Afterward, the patient presented with a mass that increased the volume of the left side of the nasogenian region and raised the nasal ala but was asymptomatic, without changes in color but with a firm consistency. Intraorally, the patient presented an increased volume in the vestibular furrow and palatal region from the upper lateral left incisor to the molar area on the same side. The mass was the same color as the adjacent mucosa, did not result in tooth mobility, and was also hard and firm.

The imaging studies showed an osteolytic lesion covering the entire left maxillary and zygomatic parts of the same side (Fig. 1A). Biopsies showed hypodensity of the lesion, with some areas of higher density from the orbital rim to the midline jaw.

To date, there is limited information about the interaction of odontogenic tumors and immune cells. The aim of this work was to analyze the immunophenotype of the T cell subset in the tumor and peripheral blood to analyze its activation function.

Surgery.

The pathological diagnosis was $\mathrm{OF}$, and given that it was a recurrent tumor, left maxillectomy with the Weber-Ferguson approach later was performed. A containment plate 2.0 system was used to prevent depression of soft tissue, and tissue conditioner was applied immediately to encourage healing of any tissue that was damaged during surgery (Fig. 1D and E). Finally, an injury-free control was imaged (Fig. 1B).

Histopathologic findings from the OF sample.

The final pathology reported was OF, while the histopathology analysis showed proliferation of benign spindle-shaped fibroblastic cells with embedded mineralized structures, which presented as round to ovoid collections of bone with an osteoid rim. Additionally, there were particles with radiating collagen fibers, whereas there were multiple ossicles with a thick irregular collagenous rim (Fig. 1C).

Analysis of the T cell subset in the tumor and peripheral blood. 
The tumor-infiltrating immune cells were assessed in tumor cell samples and by multiparameter flow cytometry analysis (Supplementary Materials and Methods). The analysis showed that $13 \%$ of cells were immune cells (CD45 ${ }^{+}$cells, Fig. 2A), of which $29.8 \%$ were T lymphocytes (CD3 ${ }^{+}$cells, Fig. 2B). Within the $T$ cell subset, it was found that half of the cells were helper T lymphocytes (Th lymphocytes, $\mathrm{CD} 3^{+} \mathrm{CD} 4^{+}$) and the other half were cytotoxic T lymphocytes (Tc lymphocytes, $\mathrm{CD} 3^{+}, \mathrm{CD} 8^{+}$) (Fig. 2C).

The relative expression of the CD69, CD25, CTLA-4, PD-1, LAG-3, and TIM-3 molecules in T lymphocyte populations was compared by fluorescence intensity, both in tumor cells and in peripheral blood cells, as observed in the histograms of Fig. 2D-E. High expression of the activating molecule CD69 by Th and TC lymphocytes was observed; however, high expression of PD-1, which is an inhibitory molecule, was also found in Th and Tc lymphocytes infiltrating the tumor and in peripheral blood. In the tumor and peripheral blood samples, the expression of LAG-3 and TIM-3 was restricted to the population of Th lymphocytes and was mostly expressed by Tc lymphocytes. CD25 and CTLA4 expression by T cells was not observed in the tumor or peripheral blood. (Fig. 2D-E).

The high expression of CD69 and PD-1 by the T lymphocyte populations in the tumor was also seen for lymphocytes from peripheral blood. These data suggest that the cells received strong inhibitory signals, which cause $T$ cells to not respond to a specific antigen. For this reason, we decided to carry out an ex vivo assay with PBMCs stimulated with a polyclonal activator (PMA/Ion) and to evaluate the expression of these molecules by $\mathrm{T}$ lymphocytes 24 hours poststimulation (Fig. 3A-B). After activation, the cells showed higher expression of CD69 and maintained increased expression of PD-1; however, there was also expression of CTLA-4, LAG-3, and TIM-3, which are molecules that produce negative signals. This phenomenon was more marked in Tc lymphocytes than in Th lymphocytes. Interestingly, it was observed that the Th population showed expression of CD25, which is related to the proliferation of T cells, but the Tc population showed no expression of CD25.

\section{Discussion And Conclusions}

In this case report, we described for the first time the expression of inhibitory molecules (PD-1, LAG-3, and TIM-3) in the T cell subset that had not previously been described in OF and odontogenic tumors. We found high expression of the PD-1 molecule on the infiltrating T cells in OF and restricted expression of the LAG-3 and TIM-3 molecules. These findings suggest that although there were T cells in the tumor that were activated (high expression of CD69), inhibitory signals predominated that probably kept the CD $4^{+}$ and $C D 8^{+} T$ cells in an exhausted state, which perhaps prevented the immune response from inducing tumor remission.

The role of T cell exhaustion in oral OF is yet to be explored. These first findings and future studies could suggest immune checkpoint molecules as new therapeutic targets in the treatment of odontogenic tumors that could contribute to conventional therapy and management of these types of benign tumors. 
The patient presented recurrent $\mathrm{OF}$ that was treated successfully by maxillectomy. Tumor-infiltrating and peripheral blood T cells showed expression of PD-1, LAG-3, and TIM-3, suggesting an exhausted T cell response.

\section{Declarations}

\section{Ethics approval and consent to participate}

The present study followed the medical and ethics protocols of the Declaration of Helsinki, and the institutional Committees of Research, Biosecurity, and Ethics approved it (Registration No. HJM 0623/191).

\section{Consent for publication}

Written informed consent for publication of the clinical details and clinical images was obtained from the patient.

\section{Availability of data and materials}

Supplementary Materials and Methods. Detailed description of the histopathological analysis, sample collection, flow cytometry analysis, and T cell activation assay.

\section{Competing interests}

All authors declare that they do not have conflicts of interest.

\section{Funding}

This work was supported by the National Autonomous University of Mexico (UNAM: PAPIIT IN225829).

\section{Authors' contributions}

$\mathrm{CL}, \mathrm{LAM}, \mathrm{MC}, \mathrm{PAG}$ and $\mathrm{CT}$ participated in the treatment of this patient and were involved in the development of the conclusions. AG, NGN, and GD performed the experiments, MAM wrote the first draft with assistance from RP edited the final draft. All authors had read and approved the final manuscript.

\section{Acknowledgements}


Not applicable.

\section{Declaration of Competing Interest}

The authors declare that they have no known competing financial interests or personal relationships that could have appeared to influence the work reported in this paper.

\section{References}

1. El-Naggar AK, Chan JKC, Takata T, Grandis JR, Slootweg PJ. The fourth edition of the head and neck World Health Organization blue book: editors' perspectives. Hum Pathol. 2017;66:10-2.

2. Wright JM, Vered M. Update from the 4th Edition of the World Health Organization Classification of Head and Neck Tumours: Odontogenic and Maxillofacial Bone Tumors. Head Neck Pathol. 2017;11(1):68-77.

3. Chrcanovic BR, Gomez RS. Juvenile ossifying fibroma of the jaws and paranasal sinuses: a systematic review of the cases reported in the literature. Int J Oral Maxillofac Surg. 2020;49(1):28-37.

4. Chen DS, Mellman I. Oncology meets immunology: the cancer-immunity cycle. Immunity. 2013;39(1):1-10.

5. Schreiber RD, Old LJ, Smyth MJ. Cancer immunoediting: integrating immunity's roles in cancer suppression and promotion. Science. 2011;331(6024):1565-70.

6. Zappasodi R, Merghoub T, Wolchok JD. Emerging Concepts for Immune Checkpoint Blockade-Based Combination Therapies. Cancer Cell. 2018;34(4):690.

7. Barber DL, Wherry EJ, Masopust D, Zhu B, Allison JP, Sharpe AH, et al. Restoring function in exhausted CD8 T cells during chronic viral infection. Nature. 2006;439(7077):682-7.

8. Richter K, Agnellini P, Oxenius A. On the role of the inhibitory receptor LAG-3 in acute and chronic LCMV infection. Int Immunol. 2010;22(1):13-23.

9. Ferris RL, Lu B, Kane LP. Too much of a good thing? Tim-3 and TCR signaling in T cell exhaustion. J Immunol. 2014;193(4):1525-30.

10. Khaitan A, Unutmaz D. Revisiting immune exhaustion during HIV infection. Curr HIV/AIDS Rep. 2011;8(1):4-11.

\section{Figures}



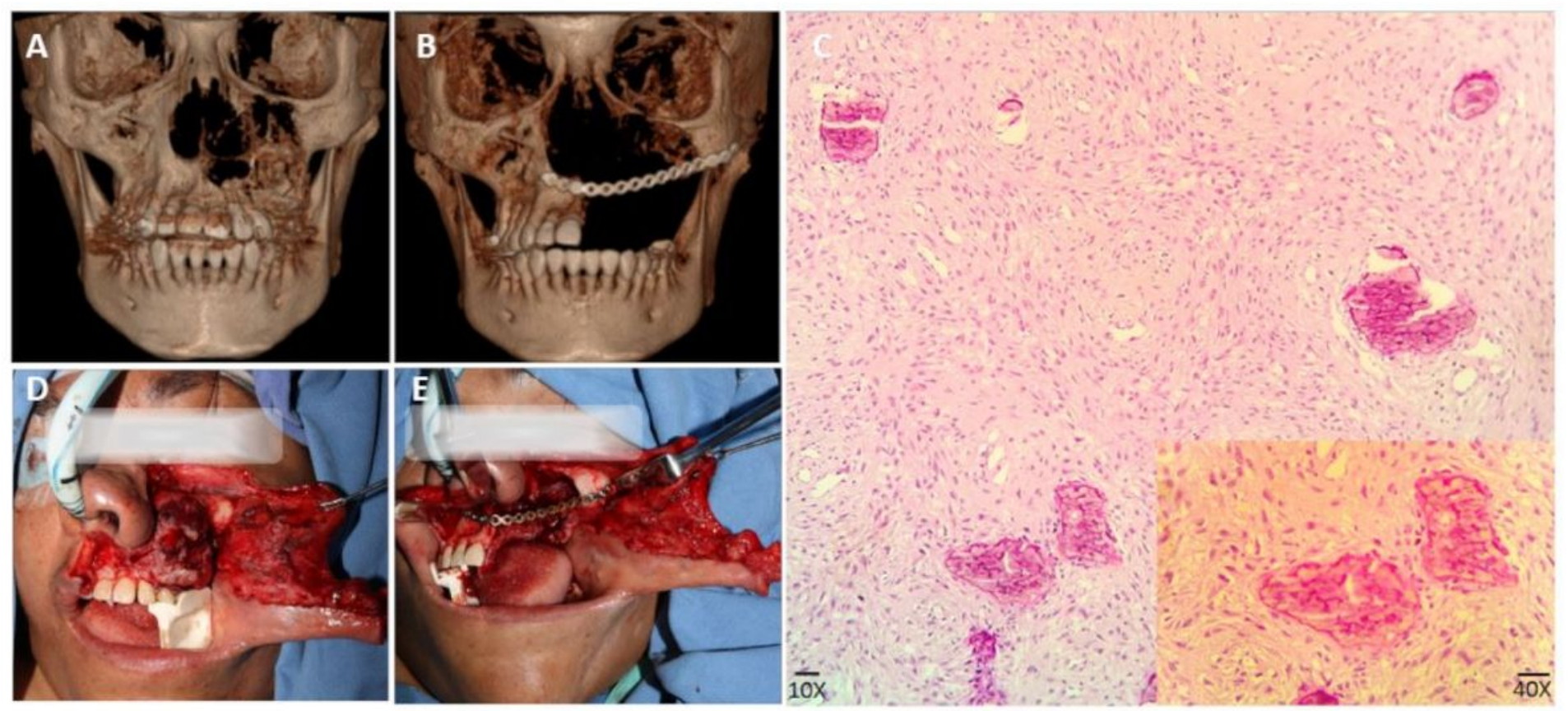

\section{Figure 1}

Clinical appearance and surgical approach of the patient. A) On computed tomography, there was tissue destruction at the level of the nasogenian region, which extended to the area surrounding the malar bone and, at the level of the oral cavity, from the region of the lateral incisor to the molar region. B) Postsurgery computed tomography. C) The histopathologic appearance of the ossifying fibroma showing highly cellular fibroblastic stroma with small calcifying spherules (hematoxylin-eosin, 10x and 40x). D) Image of the tumor before surgery. E) Image of the area postsurgery and the placement of the titanium plate used for facial reconstruction. 


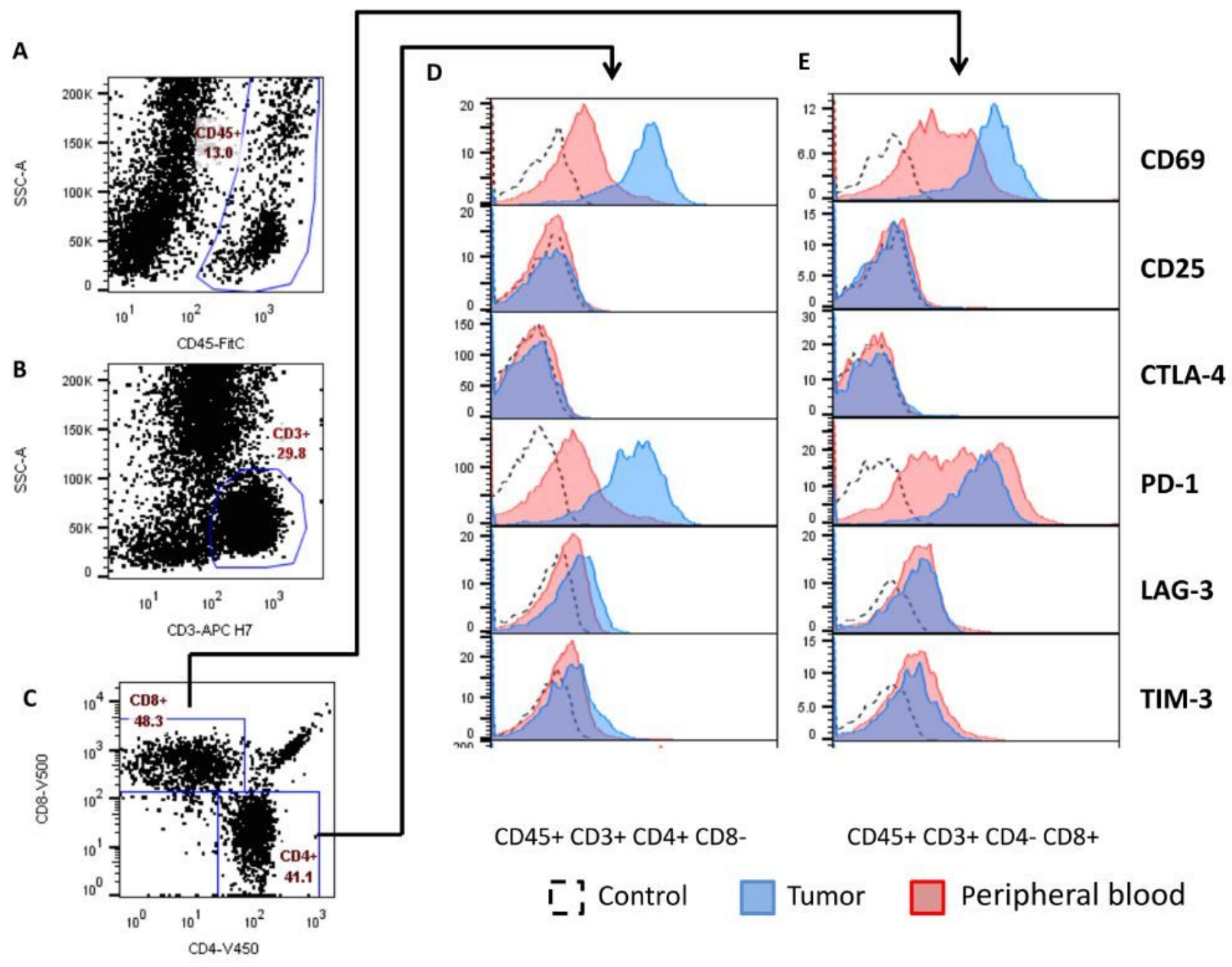

Figure 2

The phenotype of T cells from the tumor and peripheral blood as analyzed by flow cytometry. A) Dot plot of the tumor-infiltrating immune cells (CD45+), B) T lymphocytes (CD3+, CD45+), C) T helper cells (CD45+, CD3+, CD4+ CD8-) and cytotoxic T cells (CD45+, CD3+, CD4-, CD8+). (D-E) CD69, CD25, CTLA-4, PD-1, LAG-3, and TIM-3 expression in T helper cells (right) and cytotoxic T cells (left) analyzed from peripheral blood (PB) and tumor samples. The autofluorescence control defined the threshold between negative and positive. 
A

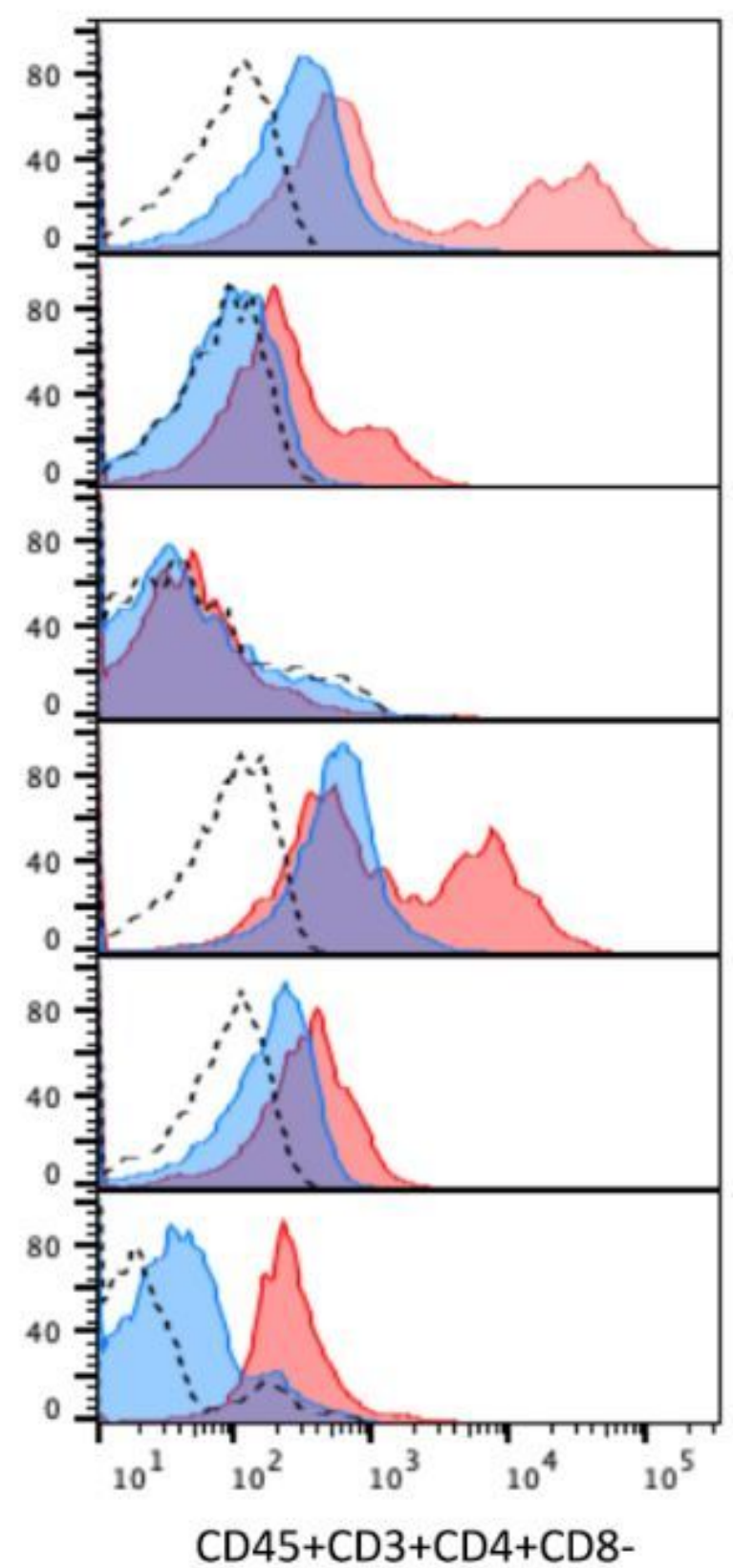

$\mathrm{CD} 45+\mathrm{CD} 3+\mathrm{CD} 4+\mathrm{CD} 8-$
B

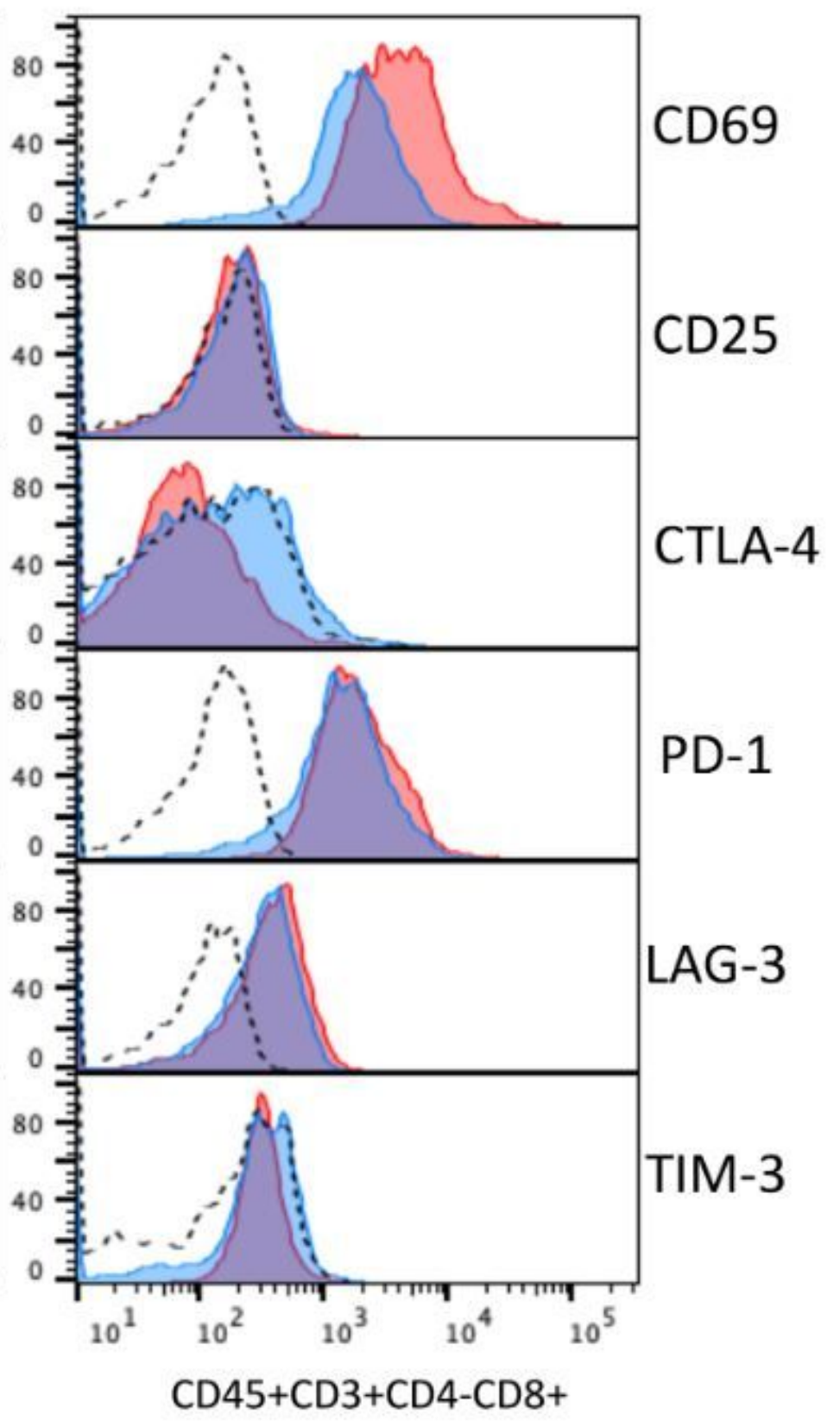

Unstimulated

PMA/Ion

\section{Figure 3}

Ex vivo activation of T cells activation from peripheral. Peripheral blood mononuclear cells (PBMCs) from the patient were stimulated with a polyclonal activator (PMA/Ion). CD69, CD25, CTLA-4, PD-1, LAG-3, and TIM-3 expression by $T$ cells poststimulation. A) CD4+ T cells and $B$ ) $C D 8+T$ cells.

\section{Supplementary Files}


This is a list of supplementary files associated with this preprint. Click to download.

- SupplementaryMaterialsandMethods.docx 